\title{
Photo Collage-based Photograph Display System on Mobile Computing Platform
}

\author{
Dongwann Kang • Sanghyun Seo
}

Received: date / Accepted: date

\begin{abstract}
In the last few decades, mobile computing platform technology has grown rapidly, as observed from smart phones that have quickly become ubiquitous. The mobile computing platform is the most widely used platform in our life today, and digital photographs captured through these devices have become routine for most people. In this study, we propose a novel artistic method for displaying photographs in mobile devices as a photo collage. Using our system, users can view a representative photograph as a collage of photographs associated with a certain event, and access each of photographs individually. To implement this, we employ centroidal Voronoi diagram (CVD) to obtain an even distribution of tiles, and use the sites as the location of tiles. We use the edge avoidance technique to prevent tiles from being located across the edges. To obtain the direction of tiles that follow near a strong edge, we employ the Edge tangent Flow field, and use the field as the directions of tiles. Finally, we search for photos that best match the tiles calculated above by using a thumbnail difference metric.
\end{abstract}

Keywords Photo Collage $\cdot$ Photomosaic $\cdot$ Mobile Platform

\section{Introduction}

Platform technologies that enable a wide range of innovative scientific works through multidisciplinary studies, such as biotechnology [20], cloud computing $[10,11]$, privacy protection cho2014,Kim2013 and security [7,23], and big

\footnotetext{
Dongwann Kang

Bournemouth University, UK

Sanghyun Seo

Division of Media Software,

Sungkyul University, Anyang-si, Kyunggi-do, Korea

Tel.: +82-31-467-8442

E-mail: shseo75@gmail.com
} 
data processing technology [5], have been developed during the last decade. Among these, the mobile computing platform technology has grown remarkably, as seen from the fact that smart phones have quickly become ubiquitous. Currently, smartphones out-sell PCs four to one [3]. Moreover, approximately half of the adult population owns a smartphone [4]. Consequently, the mobile computing platform is the most widely used platform today.

Mobile computing devices usually have built-in cameras. Consequently, taking digital photographs through these devices has become a routine activity for most people. However, the majority of the photographs captured using mobile devices every day are left in the devices without any management. To assist users manage numerous unordered photographs, several mobile applications and cloud platforms that provide automatic photograph organizing services, such as Apple Photos [1] and Google Photo [2], exist. These applications classify photographs according to events based on the time and place estimate. However, they just display the classified photographs in a sequential classic manner. This method is not appropriate for viewing an overview of photographs in mobile devices that usually have a small screen.

In this paper, we propose a novel artistic method for displaying photographs in mobile devices as a photo collage. Instead of displaying photographs sequentially, we aim to show all the photographs associated with a certain event in one screen. In our method, these photographs compose a representative photograph obtained by mixing. Therefore, users can see the representative photograph as a collage, and view each of the photographs of the event that are associated with the representative photograph. This method is not only an effective way for displaying many photographs associated with a certain event on a small screen at once, but also provides an artistic and entertaining way to experience the photographs.

The rest of this paper is organized as follows. In Section 2, we provide an overview of studies related to photo collage and collage-based stylizations. We present the details of the proposed photo collage-based photograph display system in Section 3. The implementation and results of this method are described in Section 4. Finally, we conclude by summarizing our ideas and discuss future work in Section 5.

\section{Related work}

In the last two decades, computer graphics researchers have studied photo collage techniques extensively. The first approach to photo collage was the photomosaics of Silvers and Hawley [26]. As shown in Figure 1(a), the photomosaic technique generates a mosaic image of grids composed of small photo tiles. The photo tile that is most similar in color to the inside of the grid is chosen as the tile of the grid from the photo tile database. This technique produces a resulting image that places the given specific photo tiles evenly without gaps. However, the result depends on the size and variety of database. Therefore, depending on the database, important features that must be preserved in 


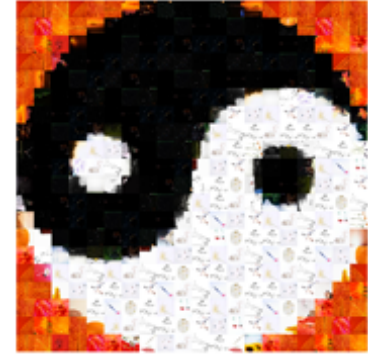

(a) Photomosaic [26]

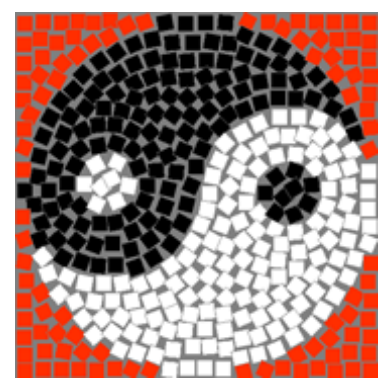

(b) Tile mosaic [9]

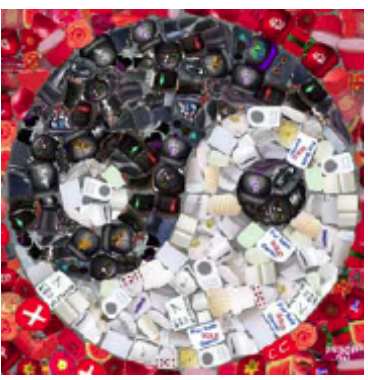

(c) JIM [18]

Fig. 1 Comparison of different collage algorithms.

the result, such as strong edges, cannot be guaranteed. Finkelstein and Range [29] proposed an image mosaic method that uses a collection of small images arranged to represent a large one. In their method, color correction was used to generate a result similar to the original input image. To measure the effectiveness of these algorithms, Tran [28] proposed a measure that considers similarity to the original input image, granularity and variety of individual photo tiles, and the running time cost.

Hausner [9] proposed a method of mosaic that provided a result close to the traditional tile mosaics (Figure 1(b)). He suggested a tile locating method using CVD [21] and edge avoidance techniques to preserve the important edges. We employ these techniques in the proposed method to generate the main layer of tiles.

Kim and Fellacini [18] proposed Jigsaw Image Mosaics (Figure 1(c)), an extension of the photomosaic method. This method locates arbitrary shapes of tiles where images are printed inside by minimizing an energy function, which is designed to return lower values when tiles are located densely without overlap. Thus, the gap and overlap between tiles is reduced in the result. This method is useful for representing specific image with small photographic tiles of arbitrary shapes. However, it is not suitable for our purpose, which targets ordinary rectangular photographs.

In the photomosaic algorithm, the search for the best matching photo tiles from database constitutes the main bottleneck. Several studies have focused on enhancing the performance of searching by using a programmable GPU. Kang et al. [16] proposed a parallel framework for fast photomosaic. They constructed a structure of vertices to represent the grids of the photomosaic, and searched for the best matching photo tiles from the database that was stored on texture using shaders. They achieved interactive time performance for photomosaic using a database that consisted of thousands of photo tiles. Choi et al. [6] also proposed a real-time approach that generates a photomosaic video. They employed genetic feature selection for building an optimized image set and GPU-accelerated pattern searching of CUDA for minimizing computation cost. On the other hand, Markuš et al. [22] proposed an image mosaic method that exhibited large performance speed-up by employing a decision tree to 
decide the most appropriate photo tile using a pre-determined codebook for each cell. Their implementation is capable of processing video streams in real time.

Extensions of the photomosaic algorithm have been developed for various fields. A stackable mosaic method [15] was proposed for use in mobile device applications. In this method, various approaches suited for mobile devices were employed to overcome the limitation of resource and computation performance. The database was optimized to reduce its size, and photographs of rotatable objects were used for tiles to expand the database without occupying resource memory. Kang and Seo [14] developed a mobile-based photo collage album application. They focused on Hockney's collage style, and extracted feature points from a sequence of photographs taken by interval shots. The photographs were deformed by using extracted feature points, and finally, a patchwork was generated by taking photo tiles from the deformed photographs. Photomosaic can also be incorporated with the social networking service. In [25], the social networking context was utilized to generate a photomosaic album that enabled the selection of photographs from the albums of users' friends in the social networking service by computing the closeness of connections between users. In addition, a number of studies for various types of mosaics $[8,12,19]$ have been conducted in the non-photorealistic rendering and animation field. However, they mainly focus on stylizing mosaic and not on photo collage.

The preliminary version of this work was presented in [13], where we focused on stylizing a mosaic only. In this paper, we extend our work to focus on the display method for photographs on mobile devices and provide improved methods suitable for mobile platform with restricted resources.

\section{Photo Collage-based Photo Display System}

From a given set of photographs associated with a certain event chosen by the user, our system constructs a database for photo tiles that will be employed in the collage. From the photographs in the set, the user selects a single photograph as a representative photograph of the event. Then, our system constructs a tile collage layout that effectively and artistically expresses the image of the representative photograph. Here, each tile is a photo tile taken from the database. Because this photo tile is similar to the part of the representative photograph corresponding to the tile area, the collage result that consists of photo tiles effectively shows the representative photograph. Finally, users can enjoy the memory of a certain event through the collage of the photographs of the event that form the representative photograph. They can also view each photograph in full resolution by selecting a photo tile. 


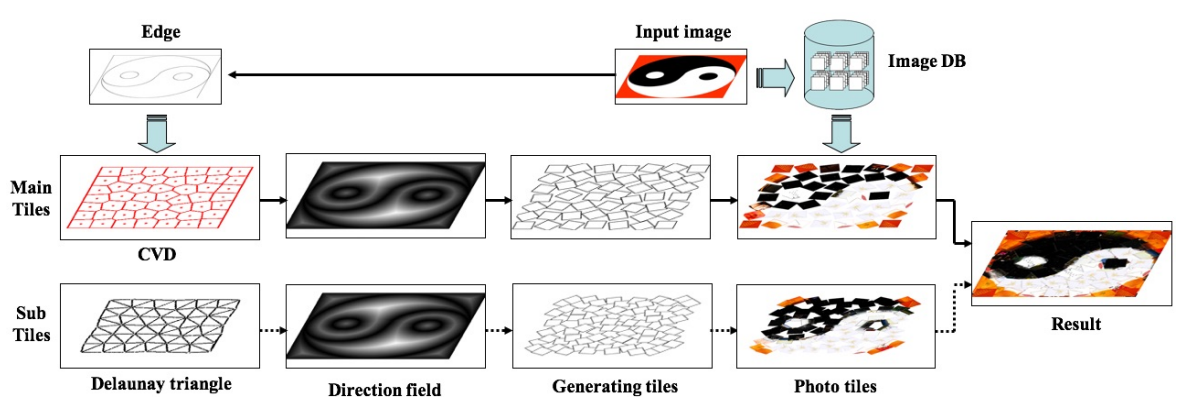

Fig. 2 System overview of the proposed method.

\subsection{System Overview}

Generally, photo tiles have to be appropriately chosen such that they express the main information of a target image, such as color and edge. This is required to obtain good quality results in the photo collage method. Consequently, it is important to increase the chance for finding the best photo tile that matches the part of the target image corresponding to the tile area by increasing the size of the database. However, in our system, the database has a very limited size because the number of the photographs in a set associated with a certain event chosen by user is usually limited to hundred. Therefore, traditional photo collage techniques, such as the photomosaic $[16,26]$, are not appropriate for our system. In order to solve this problem, we propose a novel photo collage method that enables us to obtain a reasonable quality with limited number of photo tiles. For constructing the layout of photo tiles, we use Hausner's method [9] that generates tiles of traditional mosaic, instead of using the photomosaic method that uses a grid layout. This method effectively expresses the edges of the representative photograph by locating tiles to avoid the edges. Therefore, the need to express edges is decreased for photo tiles; this enables the generation of reasonable quality collage using the restricted database. Figure 2 shows the system overview of the proposed method. We present the details of our collage method in Section 3.2.

\subsection{Collage Method using Photo Tiles}

\subsubsection{Generating the Layout of Tiles}

We use uniform-size square tiles in our system. If the distribution of tiles is not even, the tiles may overlap in the areas where they are located densely. On the contrary, the areas with sparsely located tiles will have gaps between tiles. Therefore, tiles need to be distributed evenly to prevent overlap and gaps. In stylization studies, the CVD, a special type of Voronoi diagram, was used to obtain an even distribution of points in Pointillism [24] and tiles in traditional 


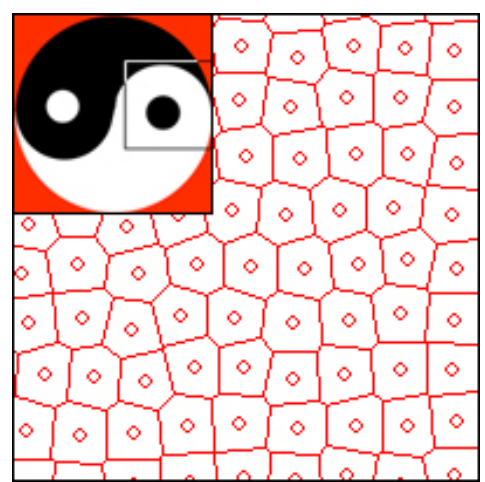

(a) CVD before the edge avoidance

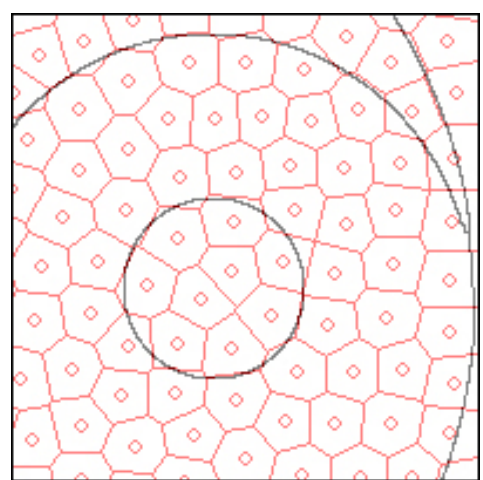

(b) CVD after the edge avoidance

Fig. 3 CVD and edge avoidance method. Red circles indicate the site of each Voronoi cell and red lines indicate the boundaries of Voronoi cells.

mosaic [9]. In this study, to get an even distribution of tiles, we employ CVD using Lloyd's method [21].

To compute the CVD, we first randomly spread seed points on a canvas whose size corresponds to that of the representative photograph, $I$. The number of seed points depends on the size of each tile. We use uniform-size square tiles, and thus can calculate the approximate number of whole tiles by using the size of each tile and overall area of the canvas.

$$
N=\frac{\delta \times w \times h}{s^{2}}(\delta \leq 1)
$$

Here, $N$ is the number of tiles, $s$ is the user-defined size of tiles, $\delta$ is a constant value for controlling the gap between tiles, and $w$ and $h$ are the width and height of the canvas, respectively. A value of $\delta=1$ implies that the tiles cover the entire area of the canvas. However, because it is almost impossible to achieve this without overlapping or creating a gap with uniform-size square tiles that have their own direction, $\delta$ must be assigned a value less than 1 . In our system, we used 0.7 as the value of $\delta$.

After locating $N$ seed points on the canvas, we compute the Voronoi diagram by using the seed points as sites. Then, we move each site to the centroid of each Voronoi cell, and re-compute the Voronoi diagram. By performing this step repeatedly, we finally obtain CVD, which has an approximately uniform distribution of cells. Consequently, we can obtain a uniform distribution of tiles by locating them on the sites of the CVD.

CVD is useful for obtaining an even distribution of tiles, but does not guarantee the location of tiles that express the edges of $I$. As mentioned in Section 3.1, to obtain a collage of reasonable quality with a limited database, tiles have to express edges of I by itself. Thus, tiles must not be located across the edges. To implement this, we employ the edge avoidance method used in [9]. Before computing CVD, we extract the edge from I, and overlay it on the canvas. Then, we enlarge the width of the edge up to the size of a tile. 


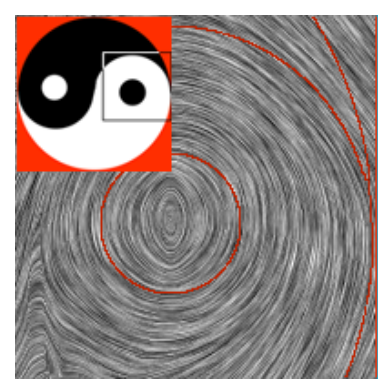

(a) ETF field

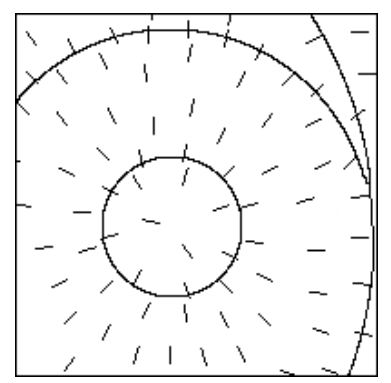

(b) The direction of tiles

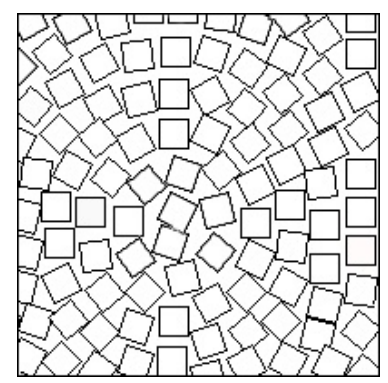

(c) Tiles with rotation

Fig. 4 ETF field and the direction of tiles obtained from ETF. ETF field (a) shows the edge flow obtained from an input image. We obtain the direction of a tile from the position on ETF field that corresponds to the site of the tile.

When we calculate the centroid of each cell while computing CVD, we do not count the enlarged edge area. Thus, we obtain the sites of CVD while avoiding edges. Figure 3 shows the CVD results before and after implementing the edge avoidance method.

Locating tiles with edge avoidance is not sufficient to express the edge of $I$; tiles must also be aligned along the direction of the edge. To achieve this, we calculate the direction of each tile by using the Edge Tangent Flow (ETF) field [17]. By computing ETF on $I$, we obtain flows that follow the near strong edges. We use ETF on the site of each cell to obtain the direction of the tile corresponding to the cell. Figure 4 shows ETF field and the direction of tiles obtained using ETF.

Finally, we draw squares of size $s$ on the sites of CVD, and rotate each of them to flow in the direction obtained through ETF.

\subsubsection{Searching Best Matching Photo Tiles}

Hausner [9] rendered tiles by filling each tile with the color sampled from $I$. In our method, we aim to overlay photo tiles from the database on the surface of tiles drawn in Section 3.2.1. To express image $I$ through photo tiles, a method is required for finding the photograph in the database that is most similar to the part of $I$ corresponding to the tile area. In this paper, we propose a method that finds the best matching photo tiles in a cost-effective manner.

To calculate the similarity between two images, the summation of color differences between corresponding pixels in the images is naively used. Before calculating this, we resize all the images in the database to a square of predefined size. This method is employed in our study to find the best matching photo tiles from the database. However, calculating color differences on every pixel is not cost-effective. Moreover, mobile platforms on which our system is executed usually have limited computing power, hence, necessitating a costeffective approach. In this study, we propose a method that searches for the best matching photo tile based on the difference between thumbnails. 


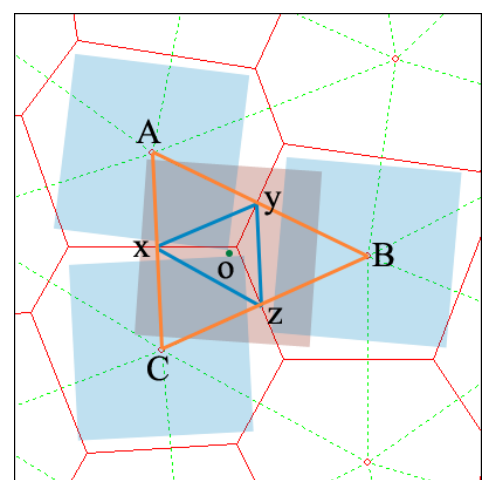

(a) Centroid of the $\triangle A B C$ and $\triangle x y z$

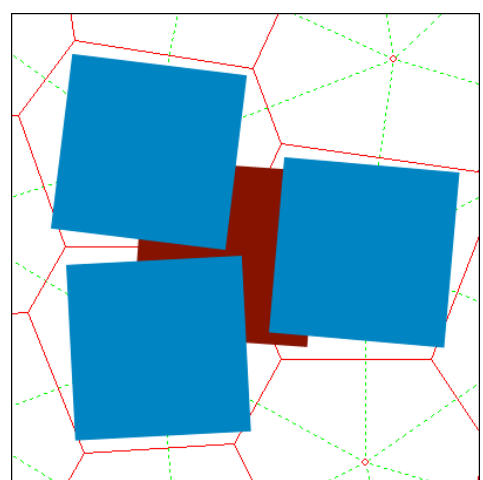

(b) Location of the sub tile

Fig. 5 Locating sub tiles using Delaunay triangulation.

The thumbnail corresponding to the $k$-th tile on the canvas, $t^{k}(k<N)$, consists of $n \times n$ grids. We divide $I$ 's sub image, $I^{k}$, which corresponds to the area of the $k$-th tile, into $n \times n$ grids. We then assign the average color of $I_{i, j}^{k}$ to a grid of the thumbnail, $t_{i, j}^{k}(i, j<n)$. Likewise, for the $m$-th photo tile in the database, $P^{m}$, we generate a thumbnail with $n \times n$ grids, $p^{m}$, which consists of average colors sampled from $P^{m}$. Instead of calculating pixel differences between $I^{k}$ and $P^{m}$, we calculate thumbnail difference between $t^{k}$ and $p^{m}$ using the following equation.

$$
D\left(t^{k}, p^{m}\right)=\sum_{i} \sum_{j}\left|t_{i, j}^{k}-p_{i, j}^{m}\right|
$$

We used the $L_{1}$ distance instead of $L_{2}$ distance to calculate the color differences of each component in rgb to achieve more cost efficiency on the mobile platform. In this study, we used 4 as the value of $n$ that has the lowest thumbnail difference. Then, we map the best matching photo tile as a texture onto the surface of the tile. To enhance the searching performance, we prepared the thumbnails of every photographs in the database before searching.

Choosing the photo tile that has the lowest difference does not always provide the best results. For instance, the same photo tiles can be chosen from the database for near low contrast regions in $I$. The resulting collage gives a monotonous feeling because of duplicated tiles. To prevent this, we excluded the photo tiles selected in neighboring tiles from the selection. We defined the neighboring tiles as the tiles which were located within pre-defined radius from a tile.

As mentioned in Section 3.1, the quality of collage depends on the size of the database. To expand the database without inserting new photo tiles, we used rotation operation in units of $90^{\circ}$ and mirror operation. Consequently, we were able to expand the database by as much as eightfold. 


\subsection{Sub Tiles}

When we calculate the number of total tiles in Section 3.2.1, we used 0.7 as the value of $\delta$. Therefore, only $70 \%$ of the total area is filled with tiles. This means that approximately a third of $I$ is filled with gaps. Because equation 1 does not consider tile overlapping, the real amount of thrown away information in $I$ is more than that.

To prevent this, we add one more tile layer, called sub tiles, under the tiles generated in Section 3.2.1, which are termed main tiles. The sub tiles are located between main tiles to fill the gaps. Therefore, a different approach is needed to locate the positions of sub tiles alongside the main tiles.

Cells are adjacent to each other in the Voronoi diagram created using the position of the main tile as the site. This means that the tiles corresponding to the cells are adjacent. As the gaps between tiles occur in areas where the main tiles are adjacent to each other, sub tiles must be positioned such that they cover the cell boundaries of the Voronoi diagram. With this knowledge, we locate sub tiles by using Delaunay triangulation.

Points $A, B$, and $C$ in Figure 5(a) represent the sites of three adjacent cells in CVD. The main tiles indicated by blue squares in the figure are located in these points. As shown in the figure, there are gaps between the tiles at the boundary of the three adjacent cells. The points $x, y$, and $z$, which divide each boundary into two equal pieces indicate the area where the gaps occur. The centroid of the blue triangle, $\triangle x y z$, is located in balance between $x, y$, and $z$. If the sub tile is located at point $o$, the gaps between the three main tiles are filled with sub tiles. Here, $o$ is the centroid of $\triangle x y z$, and corresponds to the centroid of $\triangle A B C$, which is the Delaunay triangle made by the site of CVD as shown in figure. Therefore, it is possible to use the centroid of the Delaunay triangle for each site as the location of the sub tile. The Delaunay triangle is the dual of the Voronoi diagram, and hence it is possible to obtain the Delaunay triangle from the Voronoi diagram and vice versa. Therefore, it is easy to calculate the location of sub tiles using the location of the main tiles.

The direction and size of sub tiles is calculated in a manner similar to that used for main tiles. The procedure for searching for the best matching sub tile is also similar to that used for main tiles. However, sub tiles are located under the main tiles, and so the parts of sub tiles that are hidden by main tiles do not need to be calculated for the thumbnail difference. In thumbnail difference calculation, we exclude the parts of thumbnail that correspond to the parts of sub tile that are entirely hidden by main tiles.

\section{Results}

We implemented the proposed photo collage-based photograph display system using OpenGL ES 2.0 [27] on Apple iPad's iOS environment.

Figure 6 shows the results of the photo collage technique proposed in this paper. As shown in figure, while a photo collage that uses only main tiles 


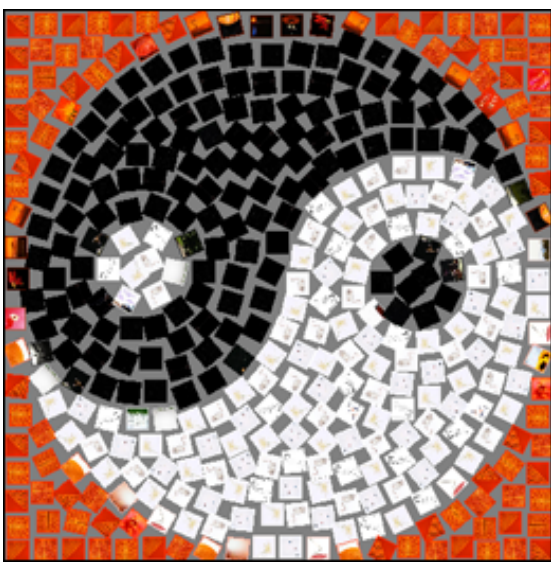

(a) Photo collage with main tiles

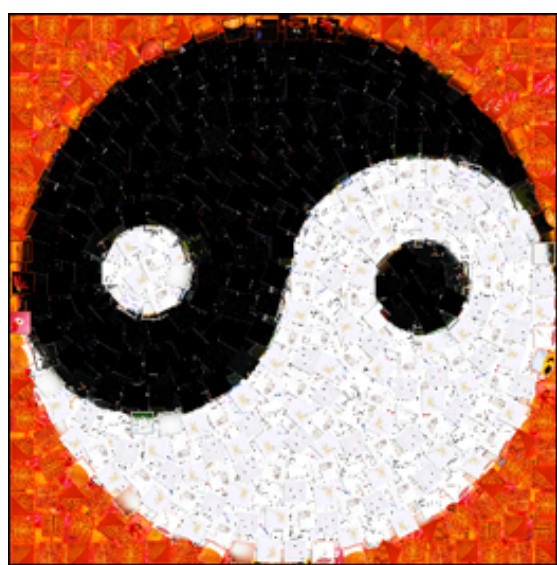

(b) Photo collage with main and sub Tiles

Fig. 6 Proposed Photo Collage Method.

(Figure 6(a)) has gaps between the tiles, a collage that uses both main and sub tiles (Figure 6(b)) effectively fills the entire area of the canvas with photo tiles. In the collage results obtained using the proposed method, the representative photograph is effectively represented while important information is preserved. Meanwhile, the photograph of each photo tile is also identified individually.

Figure 7 shows the results for different tile sizes. As shown in Figure 7(b) and $7(\mathrm{c})$, despite using tiles that are larger and less in number, important information in the representative photograph is reasonably maintained.

Figure 8 shows our system as viewed on an Apple iPad. First, the user chooses a set of photographs associated with a particular event. Then, our system builds the database of photo tiles. From the photographs of the event, the user selects a representative photograph (Figure 8(a)). Our system displays the representative photograph on screen (Figure $8(\mathrm{~b})$ ), and constructs a photo collage by using the method proposed in Section 3.2 (Figure 8(c)). Once the collage is displayed, the user can select any photo tile which is then displayed on the center of the screen (Figure 8(d)-(f)).

Figure 9 shows the proposed collage method applied to various cases.

\section{Conclusion}

In this paper, we proposed a novel artistic method for displaying photographs in mobile devices as a photo collage. Instead of displaying photographs sequentially, our system showed all the photographs associated with a certain event on a single screen. Taken together, these photographs depict a representative photograph. The user can view the representative photograph as a collage and the associated photographs individually.

To implement this application, we employed CVD to obtain an even distribution of tiles, and used the sites as the location of tiles. We used the edge 


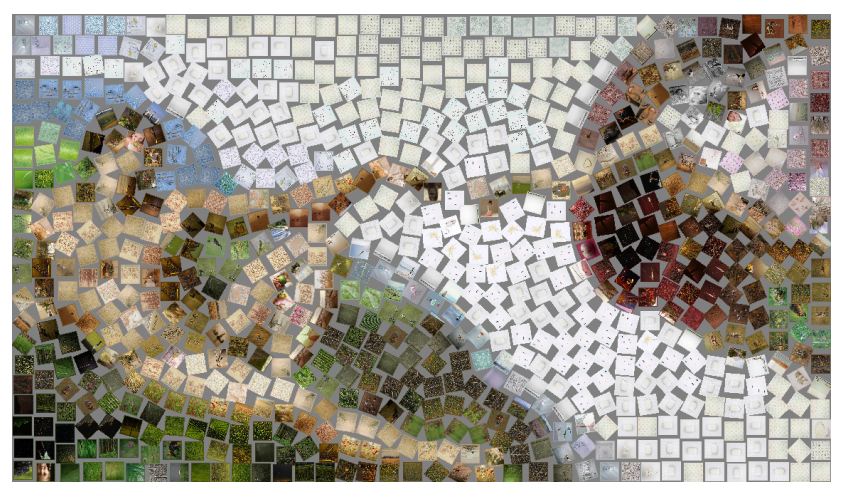

(a) Using main tiles only

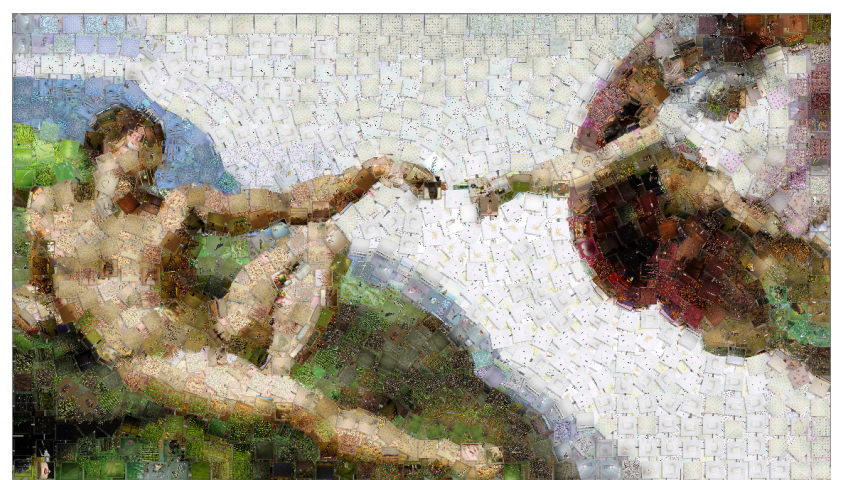

(b) Using both main and sub tiles

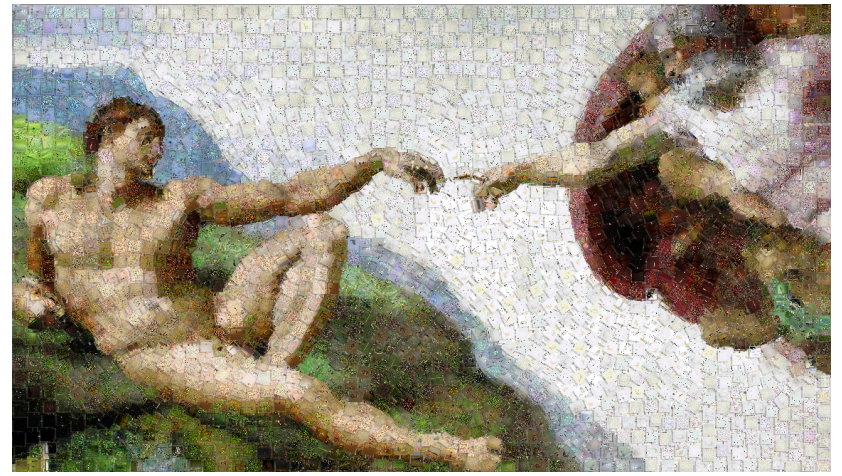

(c) Using smaller tiles than (b)

Fig. 7 Results of photo collage applied to The Creation of Adam.

avoidance technique to prevent tiles from being positioned across the edges. We employed ETF to obtain the direction of tiles that follow a near strong edge; ETF fields indicated the directions of tiles. Finally, we searched for the best matching photo tile for each tile using a thumbnail difference metric. 


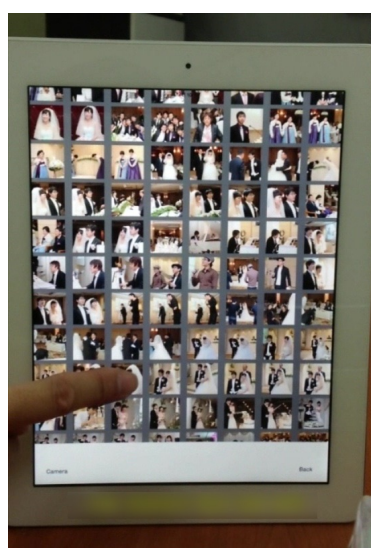

(a)

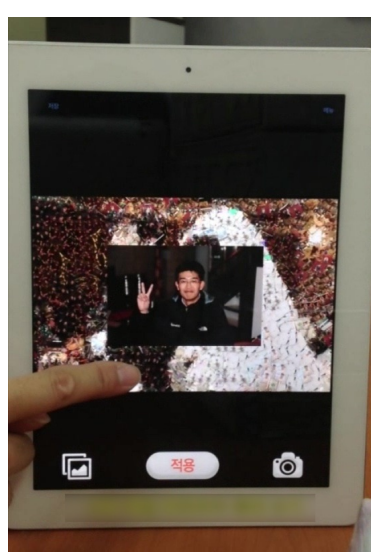

(d)

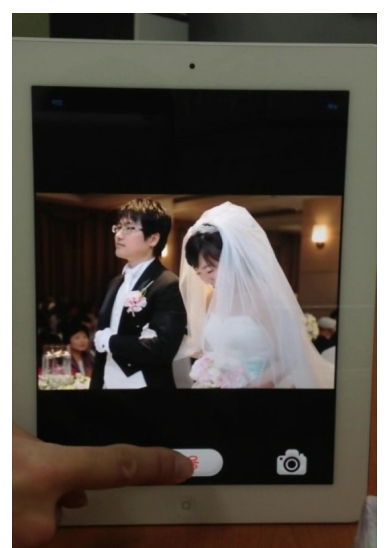

(b)

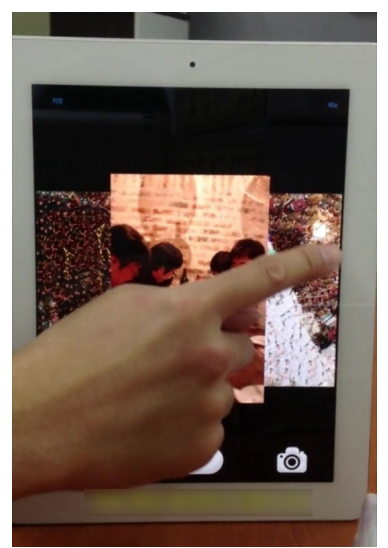

(e)

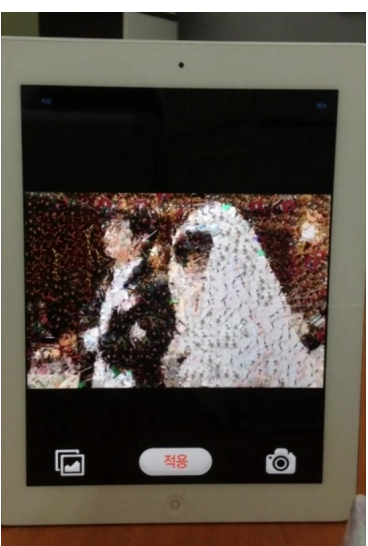

(c)

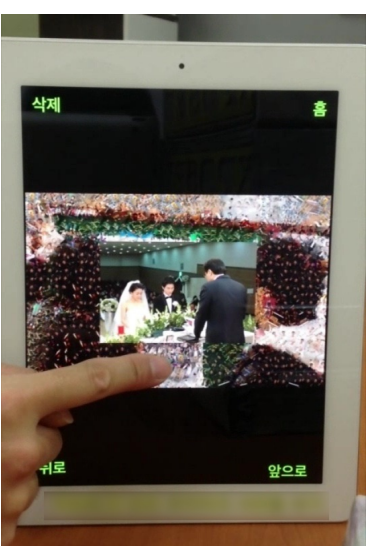

(f)

Fig. 8 Proposed photograph display system launched on Apple iPad.

In our future work, we will enhance the searching performance. Several acceleration techniques which were used in previous studies [Kang11,Choi14,Markus2015] may be utilized to enhance the performance.

\section{Acknowledgment}

This research was supported by Basic Science Research Program through the National Research Foundation of Korea(NRF) funded by the Ministry of Education, Science and Technology(NRF-2013R1A1A2061611).

\section{References}

1. Apple photos. URL https://support.apple.com/photos 
2. Google photos. URL https://photos.google.com

3. Idc worldwide quarterly mobile phone tracker, january 27,2011

4. Smartphone data are based on a pew research center survey conducted june 10-july 12 , 2015; all other data are from a march 17-april 12, 2015 survey.

5. Chen, B.W., He, X., Ji, W., Rho, S., Kung, S.Y.: Large-scale image colorization based on divide-and-conquer support vector machines. The Journal of Supercomputing 72(8), 2942-2961 (2016). DOI 10.1007/s11227-015-1414-z. URL http://dx.doi.org/10.1007/s11227-015-1414-z

6. Choi, Y.S., Jung, S., Kim, J.W., Koo, B.K.: Real-time video photomosaics with optimized image set and gpu. Journal of Real-Time Image Processing 9(3), 569-578 (2014). DOI 10.1007/s11554-013-0384-8. URL http://dx.doi.org/10.1007/s11554-013-0384-8

7. Elmisery, A.M., Rho, S., Botvich, D.: Privacy-enhanced middleware for locationbased sub-community discovery in implicit social groups. The Journal of Supercomputing $\mathbf{7 2}(1), 247-274$ (2016). DOI $10.1007 / \mathrm{s} 11227-015-1574-\mathrm{x}$. URL http://dx.doi.org/10.1007/s11227-015-1574-x

8. Han, M., Kang, D., Yoon, K.: Efficient paper mosaic rendering on mobile devices based on position-based tiling. Journal of Real-Time Image Processing 9(3), 549-556 (2014). DOI 10.1007/s11554-013-0371-0. URL http://dx.doi.org/10.1007/s11554-013-0371-0

9. Hausner, A.: Simulating decorative mosaics. In: Proceedings of the 28 th annual conference on Computer graphics and interactive techniques, SIGGRAPH '01, pp. 573-580. ACM, New York, NY, USA (2001). DOI 10.1145/383259.383327. URL http://doi.acm.org/10.1145/383259.383327

10. Huang, T.C., Shieh, C.K., Chilamkurti, N., Tsai, M.F., Rho, S.: Architecture for speeding up program execution with cloud technology. The Journal of Supercomputing 72(9), 3601-3618 (2016). DOI 10.1007/s11227-016-1715-x. URL http://dx.doi.org/10.1007/s11227-016-1715-x

11. Jabbar, S., Naseer, K., Gohar, M., Rho, S., Chang, H.: Trust model at service layer of cloud computing for educational institutes. The Journal of Supercomputing 72(1), 58-83 (2016). DOI 10.1007/s11227-015-1488-7. URL http://dx.doi.org/10.1007/s11227015-1488-7

12. Kang, D., Ohn, Y.J., Han, M.H., Yoon, K.H.: Generation of coherent mosaic animations: enhancement and evaluation of temporal coherence. Computer Animation and Virtual World 23(3-4), 191-202 (2012)

13. Kang, D., Park, Y.S., Seo, S.H., Yoon, K.H.: Two Layer Image Tile Mosaics. In: D. Fellner, C. Hansen (eds.) Eurographics Short Papers. The Eurographics Association (2006). DOI 10.2312 /egs.20061048

14. Kang, D., Seo, S.: An artistic photographic collage on a mobile device. Multimedia Tools and Applications 75(22), 14,959-14,970 (2016). DOI 10.1007/s11042-014-2316-4. URL http://dx.doi.org/10.1007/s11042-014-2316-4

15. Kang, D., Seo, S., Ryoo, S., Yoon, K.: A study on stackable mosaic generation for mobile devices. Multimedia Tools and Applications 63, 145-159 (2012). DOI 10.1007/s11042012-1065-5. URL http://liris.cnrs.fr/publis/?id=5654. Online-First

16. Kang, D., Seo, S.H., Ryoo, S.T., Yoon, K.H.: A parallel framework for fast photomosaics. IEICE Transactions on Information and Systems 94-D(10), 2036-2042 (2011). DOI http://dx.doi.org/10.1587/transinf.E94.D.2036

17. Kang, H., Lee, S., Chui, C.K.: Coherent line drawing. In: Proceedings of the 5th International Symposium on Non-photorealistic Animation and Rendering, NPAR '07, pp. 43-50. ACM, New York, NY, USA (2007). DOI 10.1145/1274871.1274878. URL http://doi.acm.org/10.1145/1274871.1274878

18. Kim, J., Pellacini, F.: Jigsaw image mosaics. ACM Trans. Graph. 21, 657-664 (2002). DOI http://doi.acm.org/10.1145/566654.566633

19. Kim, S., Kang, D., Yoon, K.: Human-friendly stylization of video content using simulated colored paper mosaics. New Review of Hypermedia and Multimedia 22(3), 270-285 (2016). DOI 10.1080/13614568.2016.1152320. URL http://dx.doi.org/10.1080/13614568.2016.1152320

20. Kim, S.S.: Mutual authentication scheme between biosensor device and data manager in healthcare environment. The Journal of Supercomputing 72(1), 177-184 (2016). DOI 10.1007/s11227-015-1536-3. URL http://dx.doi.org/10.1007/s11227-015-1536-3 
21. Lloyd, S.: Least square quantization in pcm. IEEE Transaction on Information Theory 28(2), 129-137 (1982)

22. Markuš, N., Fratarcangeli, M., Pandžić, I.S., Ahlberg, J.: Fast rendering of image mosaics and ascii art. Comput. Graph. Forum 34(6), 251-261 (2015). DOI 10.1111/cgf.12597. URL http://dx.doi.org/10.1111/cgf.12597

23. Muhammad, K., Sajjad, M., Mehmood, I., Rho, S., Baik, S.W.: A novel magic lsb substitution method (m-lsb-sm) using multi-level encryption and achromatic component of an image. Multimedia Tools and Applications 75(22), 14,867-14,893 (2016). DOI 10.1007/s11042-015-2671-9. URL http://dx.doi.org/10.1007/s11042-015-2671-9

24. Secord, A.: Weighted voronoi stippling. In: Proceedings of the $2 \mathrm{Nd}$ International Symposium on Non-photorealistic Animation and Rendering, NPAR '02, pp. 37-43. ACM, New York, NY, USA (2002). DOI 10.1145/508530.508537. URL http://doi.acm.org/10.1145/508530.508537

25. Seo, S., Kang, D.: A photomosaic image generation method using photo annotation in a social network environment. Multimedia Tools and Applications 75(20), 12,831-12,841 (2016). DOI 10.1007/s11042-015-2867-z. URL http://dx.doi.org/10.1007/s11042-015$2867-\mathrm{z}$

26. Silvers, R.: Photomosaics. Henry Holt and Co., Inc., New York, NY, USA (1997)

27. Simpson, R.: Opengl es 2.0 programmable pipeline. In: ACM SIGGRAPH 2006 Courses, SIGGRAPH '06. ACM, New York, NY, USA (2006). DOI 10.1145/1185657.1185748. URL http://doi.acm.org/10.1145/1185657.1185748

28. Tran, N.: Generating photomosaics: an empirical study. In: Proceedings of the 1999 ACM symposium on Applied computing, SAC '99, pp. 105-109. ACM, New York, NY, USA (1999). DOI http://doi.acm.org/10.1145/298151.298213 

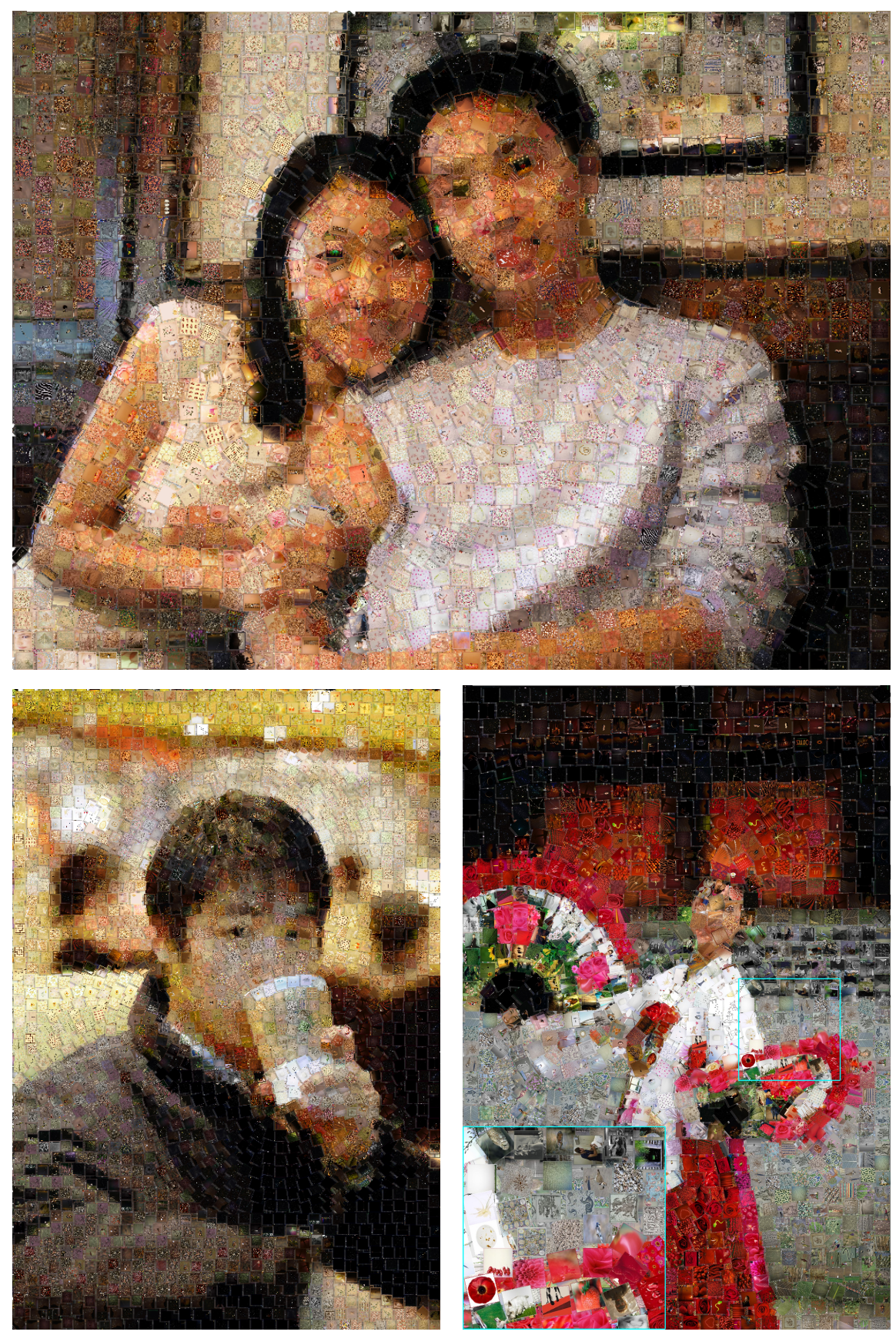

Fig. 9 Various results of our collage method. 